\title{
Increasingly translational delivery
}

The newest strategies for delivering therapeutics to specific cells and tissues are increasingly translationally promising.

$\mathrm{D}$ elivering suitable amounts of therapeutics to specific cells or tissues, and doing it safely and efficiently at appropriate times, is not unlike the transportation of goods - at minimum, one needs a suitable vehicle for the cargo, accurate mapping, a feasible route, an efficient last-mile strategy, and proof of delivery. Of course, none of these factors are near the levels of optimization of today's transportation logistics. Delivery vehicles for therapeutics are often neither robust nor entirely safe or suitable for the entire route, mapping information may be crude and changing, seemingly feasible routes are scattered with miscellaneous roadblocks (often of unknown nature), the last-mile strategy is left to the locals, and proof of delivery is not guaranteed.

Unfortunately, these analogies are only so helpful. The transportation and mapping systems are not 'alive' in ways directly comparable to fluid and cellular networks and to tissue and cellular neighbourhoods and cell compartments. Nor do we have a sufficiently deep knowledge of the rules of the body's 'roads. We can't easily re-route cargo or use a range of vehicles for the same cargo. And it may not be clear what sort of goods are needed where and when. And, in the end, the goods are most likely unwanted (and cannot be returned for re-use). Also, they are often lost en route, broken down, robbed of their essence, used for other purposes, or discarded. And when they reach their destination, it can be hard to know if they do any good, and in what ways they are actually used.

In the face of the many unknowns and challenges, many drug-delivery systems and strategies have been overengineered: larger amounts of therapeutic are delivered to compensate for the losses; alien agents, disguising coats, time bombs or decoy strategies are employed; brute-force routes are 'punctured through' to reach the intended neighbourhoods; depots are placed near target locations; or target areas are preconditioned. And yet, bodily neighbours may not be warned of an unspecific impending delivery.

Fortunately, many such strategies do work, also clinically. Unfortunately, the literature harbours plenty of delivery systems that have been excessively

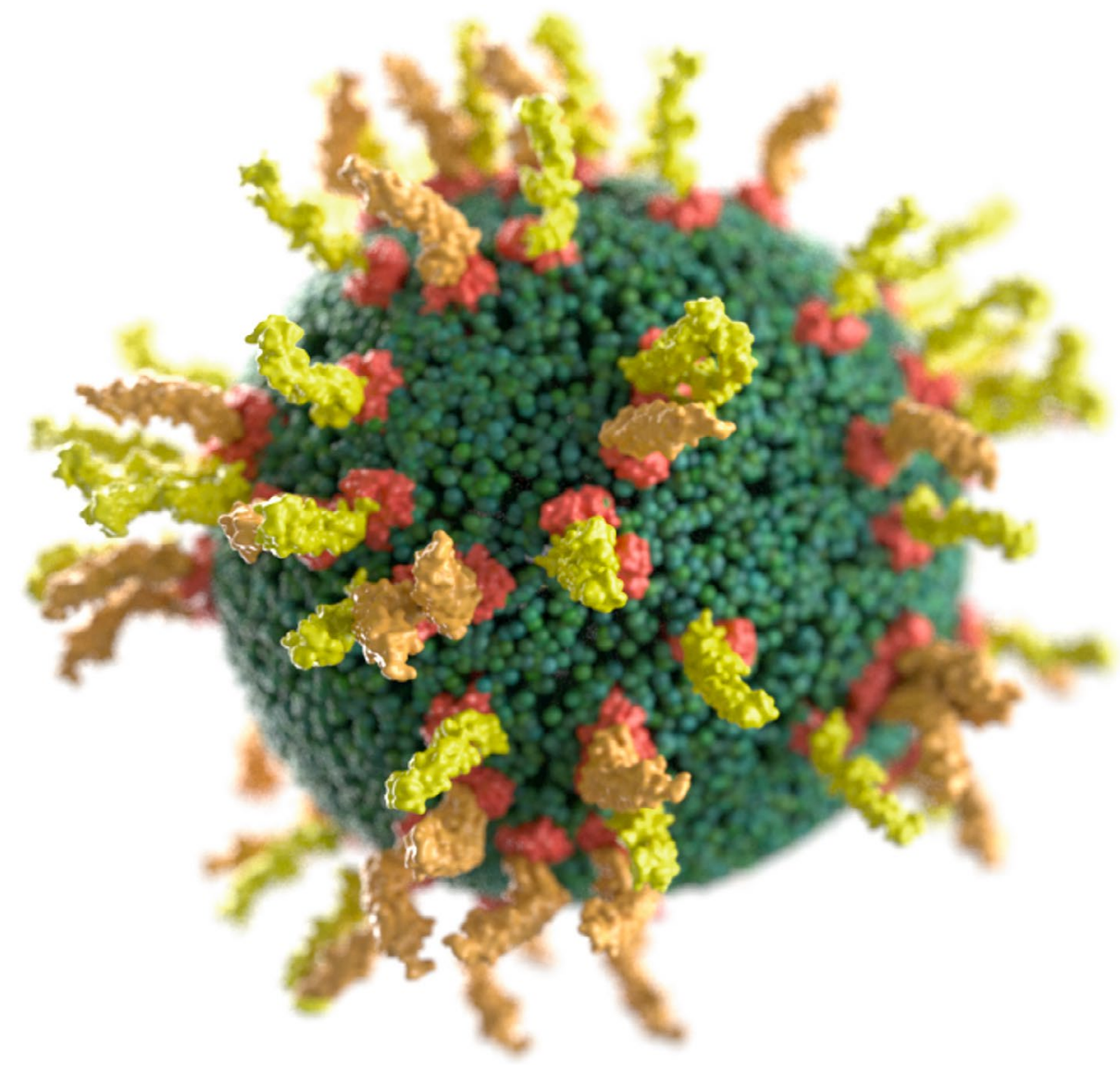

Credit: Evox Therapeutics Ltd

overengineered and that end up working only in the conditions and environments that they have been designed for: in vitro, in cell lines, ex vivo, or in small animals. This can be just fine for proof-of-principle tests. But, as the large percentage of failures in clinical trials indicate, the real world of the human body is difficult to design for. Still, when the specific target location and (ideally) the mechanism of action have been figured out, delivery strategies, routes and systems that are more targeted, specific and efficient will, in all likelihood, increase the efficacy and safety of the trialled outcomes.

But designing delivery systems for clinical translation not only requires knowledge and focus on the job-to-be-done (in particular, the type of drug, the desired functional outcomes, the tissues, cells and extracellular or intracellular structures targeted, suitable routes of delivery and, ideally, the precise biological target and the critical steps of the drug's mechanism of action), but also on the relevant constraints of the delivery job (such as pharmacokinetics, immunogenicity and specificity for the targets; as well as product-manufacturing considerations and potential patient-compliance issues). More translationally focused designs of drug-delivery systems are taking advantage of learnings ${ }^{1}$ from the development of commercialized drug-delivery products. As discussed by Ava Vargason, Aaron Anselmo and Samir Mitragotri in a Review Article in this issue of Nature Biomedical Engineering, each class of drug (small molecules, proteins and peptides, antibodies, nucleic acids and live cells) has unique delivery challenges, and benefits from different strategies or combinations of strategies for the modification of the drugs themselves (via conjugation or the alteration 
of functional groups, for example) and of their environment (for instance, by altering the $\mathrm{pH}$ of the target microenvironment or by inhibiting drug-clearance pathways). Outlining the main translationally relevant constraints early on during the design and early testing phases can only enhance the value of research and development for drug-delivery systems.

For example, when designing nanoscale delivery vehicles for small-molecule anticancer drugs, new targeting strategies often leverage translationally proven concepts to selectively increase the concentration of cytotoxic drugs in solid tumours ${ }^{2}$. In particular, pharmacokinetic and tumour-targeting considerations mean that such nanomedicines should behave stealthily when in circulation in blood, yet also stick to cell membranes so that the therapeutic can reach tumour cells. Youqing Shen and colleagues show that these two competing requirements can be met by conjugating anticancer drugs to a polyzwitterion that weakly binds to phospholipids and doesn't bind to proteins. The conjugate preferentially infiltrates tumour tissue by hitchhiking on red blood cells and by binding to endothelial cells to undergo extravasation into tumour interstitium via transcytosis.

In another Article, James Dahlman, Philip Santangelo and colleagues describe an iterative strategy for the optimization of the structure and composition of lipid nanoparticles that involves the in vivo characterization of their stability and delivery efficiency. Lipid nanoparticles developed for intramuscular delivery, such as those used in the COVID-19 mRNA vaccines, are not necessarily suitable for other delivery routes. In fact, the researchers show that nanoparticles encapsulating an mRNA encoding a broadly neutralizing antibody targeting haemagglutinin and optimized for the delivery of mRNA to the lungs via inhalation protected mice from a lethal challenge of the H1N1 subtype of influenza A virus, whereas nanoparticles previously optimized for systemic delivery did not. As noted by Rachel Yoon Kyung Chang and Hak-Kim Chan in an accompanying News \& Views, strategies for the local administration of nucleic acid therapeutics to the lungs via inhalation of nebulized or powdered formulations will become increasingly important for the treatment of respiratory diseases.

Because of their intrinsic role as intercellular shuttles, endogenous extracellular vesicles are being engineered to carry specific cargos and to present particular ligands. Samir EL Andaloussi, Joel Nordin, Dhanu Gupta, Oscar Wiklander and collaborators now show that, by screening for domains of extracellular-vesicle sorting proteins and genetically modifying vesicle-producing cells to express genetic constructs bearing oligomerized versions of optimal domains, it is possible to efficiently display two types of ligand (two decoy protein receptors for two pro-inflammatory cytokines) simultaneously on extracellular vesicles (pictured). In mice, vesicles displaying the cytokine-receptor decoys suppressed inflammation more strongly than clinically approved biologics targeting the cytokines. Functionalizing nanoscale vehicles with specific surface ligands is a rational and common approach for targeted drug delivery, and versatile methods that facilitate the control and efficiency of ligand display might be widely applicable.

Mammalian cells have also evolved to repurpose viral retroelements in their genome to shuttle specific nucleic acids to neighbouring cells. As reported in Science, Feng Zhang and colleagues found ${ }^{3}$ that the retrovirus-like protein paternally expressed gene 10 (PEG10) forms capsid-like particles packaging the protein's own mRNA, and that the mRNA cargo can be reprogrammed by flanking the chosen genes with untranslated genomic regions of Peg10. Zhang and co-authors designed a fully endogenous cell-to-cell mRNA delivery system by combining plasmids for Peg10, for the cargo RNA (bearing a desired gene, such as that for the CRISPR endonuclease Cas9, flanked by the $5^{\prime}$ and $3^{\prime}$ untranslated regions of Peg10), and for an endogenous fusogenic transmembrane protein (which can be tailored for specific cell types). Repurposing an endogenous Trojan horse as a modular delivery strategy - akin to stealthily using neighbours to make, package and deliver foreign goods among themselves, time after time - is not only potentially advantageous translationally; for some jobs, it may work fine without excess overengineering.

Published online: 6 October 2021 https://doi.org/10.1038/s41551-021-00798-7

References

1. Nat. Biomed. Eng. 2, 267-268 (2018).

2. Nat. Biomed. Eng. 3, 671-672 (2019).

3. Segel, M. et al. Science 373, 882-889 (2021). 